\title{
Orientated Growth of Hemispherical Calcium Carbonate Beneath Protein Langmuir Monolayer
}

\author{
Zhonghui Xue ${ }^{*}$, Ning Xue ${ }^{2}$ \\ ${ }^{1}$ Shanghai Publishing and Printing College, Shanghai, China \\ ${ }^{2}$ Northeastern University, Qinhuangdao, China \\ Email: *hnlgxzh@163.com
}

How to cite this paper: Xue, Z.H. and Xue, N. (2020) Orientated Growth of Hemispherical Calcium Carbonate Beneath Protein Langmuir Monolayer. Open Access Library Journal, 7: e5798.

https://doi.org/10.4236/oalib.1105798

Received: September 17, 2019

Accepted: May 6, 2020

Published: May 9, 2020

Copyright $\odot 2020$ by author(s) and Open Access Library Inc.

This work is licensed under the Creative Commons Attribution International License (CC BY 4.0).

http://creativecommons.org/licenses/by/4.0/

\begin{abstract}
Oriented calcium carbonate crystals have been grown beneath bovine serum albumin (BSA) Langmuir monolayers at the air-solution interface from supersaturated calcium bicarbonate solutions. The calcium carbonate prepared was characterized by XRD, SEM, TEM and SAED. It was found the crystal morphologies changed from amorphous calcium carbonate (ACC) to regular hemispherical calcite crystals with the prolonging of the time. These observations suggest that the process is step by step, during which the orientation of the crystalline phase was controlled by the BSA Langmuir monolayer template. These results provide new insight into the proteins-biomineral interaction, which can be extended for shape control over other minerals or inorganic-organic hybrid materials.
\end{abstract}

\section{Subject Areas}

Composite Material

\section{Keywords}

Hemispherical Calcium Carbonate

\section{Introduction}

Self-assembled two-dimensional protein layers play an important role in biomineralization processes. A well-studied example is mollusk shell nacre where the protein-rich layer is dominated by $\beta$-sheet secondary structures [1], and it is assumed that the acidic residues in this two-dimensional protein structure provide an epitaxial match to the (001) face of aragonite, which is thus favored in the crystallization of $\mathrm{CaCO}_{3}$ [2] [3]. In addition, the secondary protein structure 
seems to be associated with the $\mathrm{CaCO}_{3}$ polymorph where a change from aragonite to calcite is coupled with denaturing of the antiparallel $\beta$-sheet structure [4]. Also, the secondary structure of amelogenin was suggested to control the architecture of the hydroxyapatite phase in human enamel [5], so that changes of protein secondary structures appear to be a highly attractive additional handle for crystallization control in biomimetic mineralization approaches.

Thin membrane-like films at the air-solution interface (Langmuir monolayers) provide a more ideally flat organic surface with controllable chemical composition and molecular density [6]. These surfaces have been used as templates to direct the crystal nucleation and growth of ice, semiconductor particles, and a variety of minerals including calcium carbonate, and barium sulfate [7] [8] [9] [10]. However, they all used small surfactant molecules such as fatty acids etc. as templates to control the crystal nucleation and growth of materials. Although these surfaces can control the crystal nucleation and growth, it is disadvantageous for studying the mechanism of biomineralization.

Proteins normally have a high molecular weight, a large relative size, and a very flexible structure that can change conformation according to the experimental conditions. In addition, they can contain a high number of polar, hydrophilic groups that can make the molecule interact with the ions in the subphase. They also contain a large number of nonpolar groups that dislike being in contact with water. This combination of different groups makes a great number of proteins thermodynamically stable at the air/water interface [11] [12] [13] [14]. A lot of documents indicated the bovine serum albumin can form stable monolayers by spreading it at the air-water interface, which holds $\alpha$-helical structure [15]. Thus, BSA Langmuir monolayer with a specific secondary structure can be used as a model of biomineralization to study the nucleation and growth of crystals.

\section{Experiments}

\subsection{Materials}

Analytical grade $\mathrm{CaCO}_{3}$ was obtained form Institute of Biological Products of Tianjin (Tianjin, China). Analytical grade bovine serum albumin (Sigma, USA) was used in all experiments. BSA contains 582 amino acid residues and has a molecular weight of $67,000 \mathrm{~g} \cdot \mathrm{mol}^{-1}$. It is also well known that it has an isoelectric point at pI 4.7. Amyl alcohol was analytical purity obtained from the sigma. All solutions were prepared with triply deionized water, its conductivity was resistance of $18.2 \mathrm{M}^{\prime} \Omega \cdot \mathrm{cm}^{-1}$ and its $\mathrm{pH}$ was 7.0 .

The preparation of the protein Langmuir monolayers was performed using a commercial LB trough (KSV mini-trough, Finland) at room temperature. The pressure/area isotherms were recorded using a computer-controlled Langmuir film balance. The experimental errors for both molecular area and surface pressure values are smaller than 5\%. The monolayers deposition process at the air/solution interface was carried out using a microsyringe. The protein solutions were prepared with the deionized water, but to improve the spreading 
process a $0.05 \%(\mathrm{v} / \mathrm{v})$ solution of amyl alcohol was added in all cases [16].

Supersaturated solutions of calcium bicarbonate were prepared according to the procedures of Kitano [17]. Briefly, carbon dioxide gas was bubbled through a stirred aqueous suspension of $\mathrm{CaCO}_{3}$ for 24 hours. The suspension was then filtered and filtrate purged with $\mathrm{CO}_{2}$ gas for 0.5 hour to dissolve any remaining crystals. The resulting supersaturated solution had a $\mathrm{pH}$ of 7 . Total $\mathrm{Ca}^{2+}$ concentrations $(2.5 \mathrm{mM})$ were measured using EDTA titration. The spreading monolayers at the air-water interface were formed by spreading solutions of BSA $(1 \times$ $10^{-4} \mathrm{~mol} \cdot \mathrm{L}^{-1}$ ) on the pure water surface or Supersaturated solutions of calcium bicarbonate. A 30-min lapse time was estimated to be sufficient to equilibrate the protein monolayers before compression. We used very low compression rates, ca. $3 \mathrm{~mm} / \mathrm{min}$, which have been shown to be appropriate to obtain reproducible BSA isotherms [18].

\subsection{Crystallization under Langmuir Monolayers}

In all experiments, crystallization was governed by the slow loss of $\mathrm{CO}_{2}$ gas from unstirred supersaturated solutions according to the reaction shown in Equation (1).

$$
\mathrm{Ca}^{2+}(\mathrm{aq})+2 \mathrm{HCO}_{3}(\mathrm{aq})=\mathrm{CaCO}_{3}(\mathrm{~s})+\mathrm{CO}_{2}(\mathrm{~g})+\mathrm{H}_{2} \mathrm{O}(\mathrm{l})
$$

The prepared supersaturated calcium bicarbonate solutions were poured into a Langmuir trough (KSV mini-trough), model $611 \mathrm{M}$; maximum working area, $220 \mathrm{~cm}^{2}$ ), and the air-water interface was swept and aspirated before deposition of the surfactant solution. The surfactant solution $(10 \mu \mathrm{L})$ was carefully deposited onto the solution surface, and the monolayers were left for $30 \mathrm{~min}$ prior to compression. Pressure-area isotherms were recorded while compressing the monolayers at rates of $3 \mathrm{~mm} / \mathrm{min}$ until surface pressures corresponding to a target pressure were reached $\left(10 \mathrm{mN} \cdot \mathrm{m}^{-1}, 15 \mathrm{mN} \cdot \mathrm{m}^{-1}, 20 \mathrm{mN} \cdot \mathrm{m}^{-1}, 25 \mathrm{mN} \cdot \mathrm{m}^{-1}\right)$. Each experiment was repeated three times with the same condition. Crystals as-grown in association with the monolayers were respectively removed after $6 \mathrm{~h}$ by carefully horizontally dipping hydrophilic glass slides through the air-water interface. The crystal face growing into the solution is therefore directly deposited on the glass slide.

\subsection{Characterization of Crystal Phase and Morphology of $\mathrm{CaCO}_{3}$}

The sizes and morphologies of $\mathrm{CaCO}_{3}$ were characterized by using SEM on JSM-5600LV scanning electron microscopy (Jeol. Ltd. Japan) with operating at $30 \mathrm{kV}$. The slides supporting the crystals were mounted on copper sample stubs with conducting carbon tape and were sputter-coated with gold prior to viewing.

\section{Results and Discussion}

\subsection{The П-A Isotherms of Spreading Langmuir Monolayer on Pure Water and Calcium Carbonate Solutions}

Figure 1(a) shows the surface pressure-area isotherms of BSA spreading on a 
pure water surface as well as on the subphase of supersaturated calcium bicarbonate. BSA formed a Langmuir monolayers structure on a pure water surface as reported previously [19] [20]. When spreading BSA on the subphase of supersaturated calcium bicarbonate, on the one hand, larger changes were observed. The isotherms on the subphase of supersaturated calcium bicarbonate solution became condensed and showed a much lower initial surface pressure compared with that on pure water subphase. The limiting area was about $750 \AA^{2}$ molecule $^{-1}$ on calcium bicarbonate subphase. It is also well known that BSA has an isoelectric point at $\mathrm{pH} 4.7$, however, the $\mathrm{pH}$ value of supersaturated calcium bicarbonate solution is about 7, which made BSA Langmuir monolayers with negative charges, this result strongly electric interaction between BSA Langmuir monolayers and $\mathrm{Ca}^{2+}$ ions in the subphase. At the same time, we study the dependence of barrier position on time through the curve of time-barrier position (as shown in Figure 1(b)) when the subphase is a supersaturated calcium bicarbonate solution. It indicates that the barrier position increases with time when the surface-pressure keep at different values, in other words, the area between the two barriers gradually reduces with time, which could further account for the interaction-existing between the BSA Langmuir monolayers with $\mathrm{Ca}^{2+}$ ions in the subphase solution, which make the area of monomolecular diminish. On the other hand, although the limiting area per molecular and initial surface pressure have a larger change, the shape of surface pressure-isothermal held a same trend with onto the pure water subphase, which may indicate the BSA Langmuir monolayer keep the same secondary structure onto two subphases of pure water and supersaturation calcium carbonate.

\subsection{SEM and TEM Images}

It was observed that the almost similar morphologies of $\mathrm{CaCO}_{3}$ could be formed when it is grown in BSA Langmuir monolayers at different times, as shown by the SEM images reproduced in Figures 2(a)-(d). However, the size of the Ca$\mathrm{CO}_{3}$ particles undergoes a series of change as shown in Table 1.

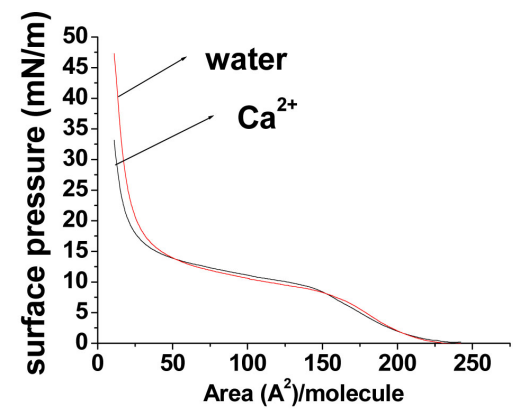

(a)

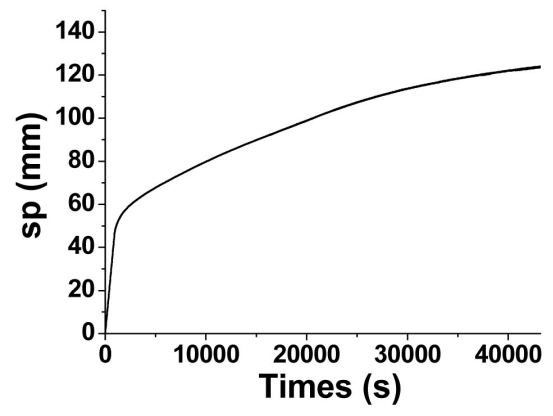

(b)

Figure 1. The surface pressure-area isotherm of BSA spreading on pure water subphase and subphase containing supersatured calcium bicarbonate (a), the barrier position-time curve of BSA Langmuir monolayers on calcium bicarbonate when compressing and keeping for $12 \mathrm{~h}(\mathrm{~b})$. 


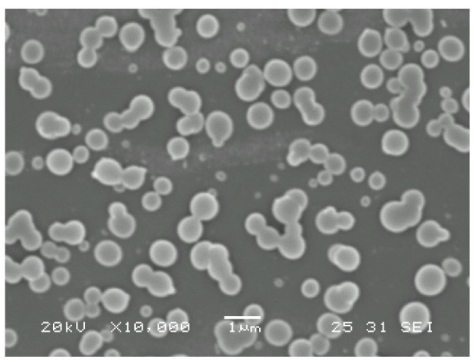

(a)

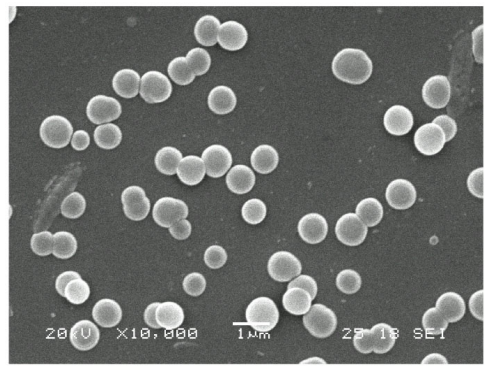

(c)

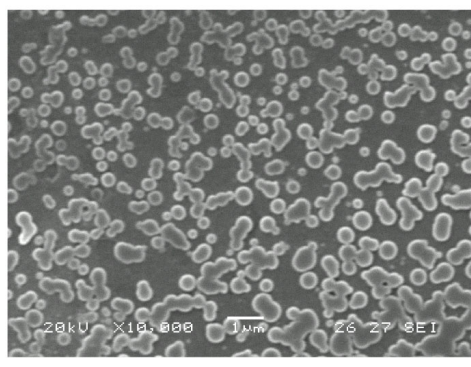

(b)

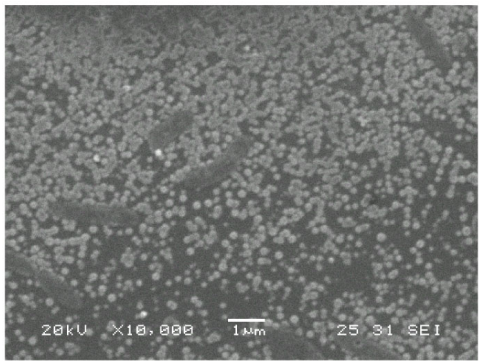

(d)

Figure 2. SEM images of $\mathrm{CaCO}_{3}$ particles obtained in presence of BSA Langmuir monolayers a denotes $8 \mathrm{~h}, \mathrm{~b}$ denotes $12 \mathrm{~h}, \mathrm{c}$ denotes $24 \mathrm{~h}$ and d denotes $48 \mathrm{~h}$, respectively.

Table 1. The size of $\mathrm{CaCO}_{3}$ particles formed beneath BSA Langmuir monolayer at different times.

\begin{tabular}{ccccc}
\hline Times (h) & 8 & 12 & 24 & 48 \\
\hline Size (nm) & 300 & 200 & 700 & 100 \\
Number & few & more & few & more \\
\hline
\end{tabular}

Simultaneously, TEM image shows that the calcium carbonate formed spherical particles or aggregates with average size about $200 \mathrm{~nm}$ after $12 \mathrm{~h}$, which is consistent with the results of SEM as shown in Figure 2(b), and the corresponding SAED indicates forming amorphous phase of calcium carbonate as shown in Figure 3(b), in this connection, the following XRD patterns give a uniform conclusion as shown in Figure 4(a). Amorphous calcium carbonate hemi-spherulites are transparent and become opaque with time. Transition of amorphous calcium carbonate hemi-spherulites into a crystalline phase was also detected from the following XRD patterns as shown in Figure 4(b), Figure 4(c).

\subsection{XRD Patterns}

Figures 4(a)-(d) show that XRD patterns of the resulting calcium carbonate at three different time. Figure 4(a) and Figure 4(b) show that the XRD pattern of calcium carbonate in the presence of BSA Langmuir monolayers, indicating that the amorphous $\mathrm{CaCO}_{3}$ was formed before $12 \mathrm{~h}$. When the crystallization time is $24 \mathrm{~h}$ and $48 \mathrm{~h}$, it could be seen that there are only one peak corresponding to calcite (104) plane of rhombohedral phase (JCPDS 86-2342) as shown in Figure 4(c) and Figure 4(d), which shows a well preferential orientation. 


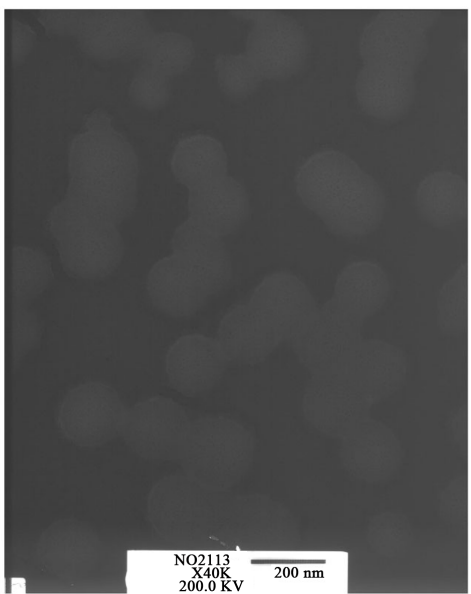

(a)

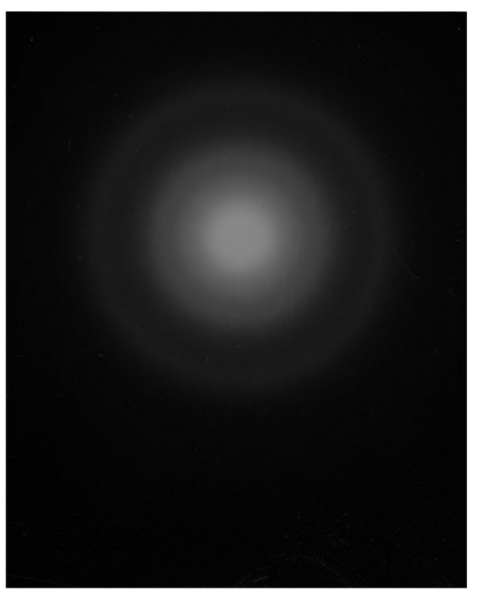

(b)

Figure 3. TEM image (a), and corresponding SAED of $\mathrm{CaCO}_{3}$ particles obtained (b) in presence of BSA Langmuir monolayers after $12 \mathrm{~h}$.

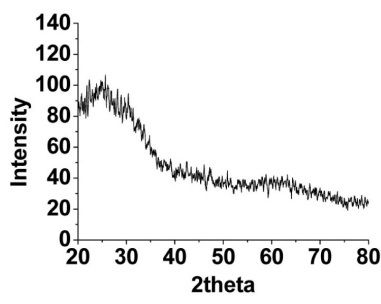

(a)

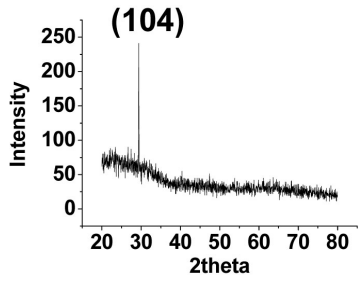

(c)

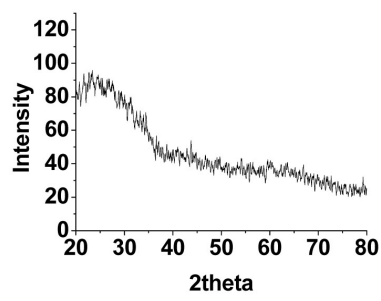

(b)

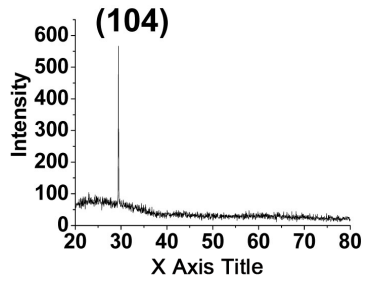

(d)

Figure 4. XRD patterns of $\mathrm{CaCO}_{3}$ in presence of BSA Langmuir monolayers at different time, $\mathrm{A}, \mathrm{B}$ and $\mathrm{C}$ denote the $\mathrm{XRD}$ patterns of $\mathrm{CaCO}_{3}$ formed at $8 \mathrm{~h}, 12 \mathrm{~h}, 24 \mathrm{~h}$ and $48 \mathrm{~h}$ respectively.

\section{Discussions}

\subsection{Molecular Recognition between the BSA and Calcite}

The results clearly shown that the BSA Langmuir monolayer could powerfully affect the nucleation and crystallization of calcium carbonate. The morphology of calcium carbonate does not at different times. With regard to the secondary structure of BSA molecular, it adopts $\alpha$-helix structure, which arrays along certain direction at the interface at the interface of air-solution. The special nucleation sites were formed, which caused the characteristic morphologies of calcium carbonate. From the viewpoint of proteins-biomineral interaction, BSA moleculars may recognize and bind to specific crystal faces, affecting growth and morphology. In the whole process of crystallization, the BSA moleculars may be 
used as a process-directing additive to control the transformation from the ACC to calcite. Generally, geometrical matches and electrostatic attraction might play an important role in the nucleation and growth of calcium carbonate.

\subsection{The Mechanism of Nucleation and Crystallization of Calcium Carbonate}

It is well known that the crystals formation involves two steps: nucleation and growth. Let us look at the general nucleation process: the constituent molecules or ions in the solution may, on collision, join into groups of two or more particles to form dimmers, trimers, tetramers, and so forth. Before the embryos can reach a critical radius, $r_{\mathrm{c}}$, they are unstable. To reach $r_{\mathrm{c}}$, an energy barrier, the so-called nucleation barrier, needs to overcome. In which way and at which number the embryos reach the critical radius is main concern. When the nucleation barrier is overcome, the second stage of the phase transition begins: growth.

In our experiments, in the initial stage of nucleation of calcium carbonate, the transformation of calcium carbonate from the unstable amorphous calcium carbonate to the most stable calcite was proved by SAED and XRD patterns. According to above results, the process of nucleation and crystallization of calcium carbonate may be as following (Scheme 1): firstly, calcium ions binding to BSA monolayer, which is a prerequisite for subsequent nucleation, then formed ACC, at last transformed into the most stable phase calcite. Since ACC formed under conditions of sequential precipitation would be the most soluble phase, a lower energy barrier is expected according to the Ostward' law [21] [22]. So it should be the first solid phase formed during the crystallization and therefore could be prevalent in biomineralization [23] [24].

Dissection of the crystallization process into several stages could make the activation energy of each step lower than that of the one-step precipitation. A multistep crystallization process is plausible, especially in a biological environment in which temporal modifications of the crystallization kinetics may be prevalent. The existence of several phases would enable organisms to control mineralization through intervention with the kinetics. By selectively interaction with the mineral at different stages during the crystal forming process, the organisms could choose to manipulate both the polymorph and the orientation of the mineral to meet specific biological requirements. Given the great diversity in the phase, morphology, and orientation of biominerals, it may be possible that complicated multistep crystallization mechanism exits [25]. The results of this paper provided direct evidence in a model system for a multistep crystallization

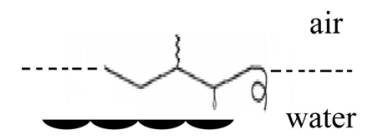

(a)

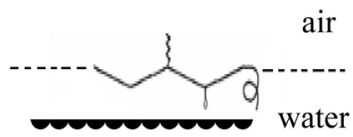

(b)

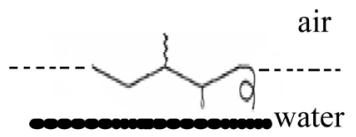

(c)

Scheme 1. Proposed mechanism for the formation of dumbbell-shaped $\mathrm{CaCO}_{3}$ particles. process that may be an important pathway for biological mineralization. This 
sequence of events stands in contrast to the prevailing view of epitaxy crystal, which is coincident to that of the calcium carbonate formed by many organisms [26] [27].

\section{Conclusion}

BSA Langmuir monolayer has played a critical role in the nucleation and crystallization of calcium carbonate. Calcium ions binding to the head groups of molecules at the interface of film-solution are a prerequisite of subsequent nucleation. Geometrical matches and electrostatic attractions between the monolayer and calcium carbonate were the main factors in determining the size and morphology of calcium carbonate formed. The observation of the transformation of calcium carbonate from ACC phase to the crystalline phase calcite provided direct evidence for the multistep-stage crystallization process in biomineralization. This work suggested a strategy for a synthesis of advanced materials under mild conditions.

\section{Conflicts of Interest}

The authors declare no conflicts of interest regarding the publication of this paper.

\section{References}

[1] Addadi, L. and Weiner, S. (1992) Control and Design Principles in Biological Mineralization. Angewandte Chemie International Edition, 31, 153-169. https://doi.org/10.1002/anie.199201531

[2] Weiner, S. and Traub, W. (1980) X-Ray Diffraction Study of the Insoluble Organic Matrix of Mollusk Shells. FEBS Letters, 111, 311-316.

https://doi.org/10.1016/0014-5793(80)80817-9

[3] Weiner, S., Traub, W. and Parker, S.B. (1984) Macromolecules in Mollusc Shells and Their Functions in Biomineralization. Philosophical Transactions of the Royal Society B, 304, 425-434.

[4] Choi, C.S. and Kim, Y.W. (2000) A Study of the Correlation between Organic Matrices and Nanocomposite Materials in Oyster Shell Formation. Biomaterials, 21, 213-222. https://doi.org/10.1016/S0142-9612(99)00120-9

[5] Batina, N., Renugopalakrishnan, V., Lavin, P.N.C., Guerrero, J.C.H., Morales, M. and Garduno-Juarez, R.J. (2002) An Atomic Force Microscopic Study of the Ultrastructure of Dental Enamel Afflicted with Amelogenesis Imperfect. Journal of Biomaterials Science Polymer Edition, 13, 337-348. https://doi.org/10.1163/156856202320176565

[6] Xue, Z.H., Hu, B.B., Dai, S.X. and Du, Z. L. (2012) Crystallization and Self-Assembly of Flowerlike Superstructures of Calcium Carbonate Regulated by Pepsin Langmuir Monolayers. Materials Chemistry and Physics, 136, 771-777.

https://doi.org/10.1016/j.matchemphys.2012.07.054

[7] Davey, R.J., Maginn, S.J., Steventon, R.B., Ellery, J.M., Murrell, A.V., Booth, J., Godwin, A.D. and Rout, J.E. (1994) Nucleation of Crystals under Langmuir Monolayers: Kinetic and Morphological Data for the Nucleation of Ice. Langmuir, 10, 1673-1675. https://doi.org/10.1021/la00018a012 
[8] Yang, J., Meldrum, F.C. and Fendler, J.H. (1995) Epitaxial Growth of Size-Quantized Cadmium Sulfide Crystals under Arachidic Acid Monolayers. The Journal of Physical Chemistry, 99, 5500-5504. https://doi.org/10.1021/j100015a037

[9] Heywood, B.R., Rajam, S. and Birchall, J.D. (1988) Controlled Crystallization of $\mathrm{CaCO}_{3}$ under Stearic Acid Monolayers. Nature, 334, 692-695.

https://doi.org/10.1038/334692a0

[10] Heywood, B.R. and Mann, S. (1992) Crystal Recognition at Inorganic-Organic Interfaces: Nucleation and Growth of Oriented $\mathrm{BaSO}_{4}$ under Compressed Langmuir Monolayers. Advanced Materials, 4, 278-282. https://doi.org/10.1002/adma.19920040407

[11] Roberts, G.G. (1990) Langmuir Blodgett Films. Plenum, New York. https://doi.org/10.1007/978-1-4899-3716-2

[12] Sánchez-González, J., Cabrerizo-Vílchez, M.A. and Gálvez-Ruiz, M.J. (2001) Interactions, Desorption and Mixing Thermodynamics in Mixed Monolayers of $\beta$-Lactoglobulin and Bovine Serum Albumin. Colloids and Surfaces B, 21, 19-27. https://doi.org/10.1016/S0927-7765(01)00180-1

[13] MacRitchie, F. (1990) Chemistry at Interface. Academic Press, San Diego.

[14] MacRitchie, F. (1997) Desorption of Protein Monolayers. Journal of Colloid and Interface Science, 61, 223-226. https://doi.org/10.1016/0021-9797(77)90384-8

[15] Lu, J.R., Su, T.J. and Thomas, R.K. (1999) Structural Conformation of Bovine Serum Albumin Layers at the Air-Water Interface Studied by Neutron Reflection. Journal of Colloid and Interface Science, 213, 426-437. https://doi.org/10.1006/jcis.1999.6157

[16] Sánchez-González, J., Cabrerizo-Vílchez, M.A. and Gálvez-Ruiz, M.J. (1999) Evaluation of the Interactions between Lipids and $\gamma$-Globulin Protein at the Air-Liquid Interface. Colloids and Surfaces B, 12, 123-138. https://doi.org/10.1016/S0927-7765(98)00069-1

[17] Kitano, Y. (1962) The Behavior of Various Inorganic Ions in the Separation of Calcium Carbonate from a Bicarbonate Solution. Bulletin of the Chemical Society of Japan, 35, 1973-1980.

[18] Sánchez-González, J., Ruiz-García, J. and Gálvez-Ruiz, M.J. (2003) Langmuir-Blodgett Films of Biopolymers: A Method to Obtain Protein Multilayers. Journal of Colloid and Interface Science, 267, 286-293. https://doi.org/10.1016/S0021-9797(03)00754-9

[19] Clark, D.C., Smith, L.J. and Wilson, D.R. (1988) A Spectroscopic Study of the Conformational Properties of Foamed Bovine Serum Albumin. Journal of Colloid and Interface Science, 121, 136-147. https://doi.org/10.1016/0021-9797(88)90416-X

[20] Owaku, K. (1989) Preparation and Characterization of Protein Langmuir-Blodgett Films. Thin Solid Films, 180, 61-64. https://doi.org/10.1016/0040-6090(89)90054-0

[21] Taylor, M.G., Simkiss, K., Greaves, G.N., Okazaki, M. and Mann, S. (1993) An X-Ray Absorption Spectroscopy Study of the Structure and Transformation of Amorphous Calcium Carbonate from Plant Cystoliths. Proceedings of the Royal Society of London. Series B, 252, 75. https://doi.org/10.1098/rspb.1993.0048

[22] Ziegler, A. (1994) Ultrastructure and Electron Spectroscopic Diffraction Analysis of the Sternal Calcium Deposits of Porcellio scaber Latr. (Isopoda, Crustacea). Journal of Structural Biology, 112, 110-116. https://doi.org/10.1006/jsbi.1994.1012

[23] Oliver, S., Kuperman, A., Coombs, N., Lough, A. and Ozin, G.A. (1995) Lamellar Aluminophosphates with Surface Patterns That Mimic Diatom and Radiolarian Microskeletons. Nature, 378, 47-50. https://doi.org/10.1038/378047a0 
[24] Aizenberg, J., Lambert, L., Addadi, L. and Weiner, S. (1996) Stabilization of Amorphous Calcium Carbonate by Specialized Macromolecules in Biological and Synthetic Precipitates. Advanced Materials, 8, 222-226.

https://doi.org/10.1002/adma.19960080307

[25] Xu, G.F., Aksay, I.A. and Groves, J.T. (2001) Continuous Crystalline Carbonate Apatite Thin Films. A Biomimetic Approach. Journal of the American Chemical Society, 123, 2196-2203. https://doi.org/10.1021/ja002537i

[26] Ruaudel-Teixier, A., Barraud, A., Belbeoch, B. and Roulliay, M. (1983) Langmuir-Blodgett Films of Pure Porphyrins. Thin Solid Films, 99, 33-40. https://doi.org/10.1016/0040-6090(83)90356-5

[27] Bermen, A., Ahn, D.J., Lio, A., Salmeron, M., Reichert, A. and Charych, D. (1995) Total Alignment of Calcite at Acidic Polydiacetylene Films: Cooperativity at the Organic-Inorganic Interface. Science, 269, 515-518.

https://doi.org/10.1126/science.269.5223.515 